\title{
The evolution of severity of paediatric COVID-19 in Singapore: Vertical transmission and multisystem inflammatory syndrome in children
}

\begin{abstract}
Dear Editor,
Coronavirus disease 2019 (COVID-19) arrived in Singapore in January 2020 as imported cases, followed by local transmission predominantly involving dormitories, with later spread within the wider community. Children still represent the minority of cases in Singapore, with around 8,000 paediatric cases as of 6 November 2021 out of a total of over 200,000 cases. Only $0.034 \%$ of children younger than 12 years old needed oxygen supplementation, intensive care, or died. ${ }^{1,2} \mathrm{~A}$ few possible reasons have been postulated: firstly, children may have more robust innate responses to viral infections; secondly, angiotensin-converting enzyme 2 receptors may be immature or less expressed in the respiratory tract of a child; and thirdly, children may have increased mucociliary clearance. ${ }^{3}$
\end{abstract}

In 2021, with the emergence of the Delta variant, rising local transmission, and children $<12$ years old remaining unvaccinated, we started to experience the fuller spectrum of paediatric SARS-CoV-2 infection. We describe the first Singapore cases known to the authors of vertically transmitted COVID-19, and multisystem inflammatory syndrome in children (MIS-C) associated with COVID-19, in the setting of a tertiary paediatric unit in National University Hospital in Singapore. The unit has about 109 paediatric inpatient beds and an intensive/high dependency care capacity of 18 beds.

Vertically transmitted COVID-19 in a newborn. In our centre, mothers with active COVID-19 infection are offered the option of rooming separately from their newborn after delivery, especially if they are deemed highly infectious by being early in illness with a low cycle threshold (CT) value. Active COVID-19 infection is defined as duration of the illness and infectivity is generally taken to be 10 days if the woman is fully vaccinated and 14 days if she is unvaccinated. Breast milk is the preferred feeding option regardless of whether the newborn is separated from the mother or rooming with her. Investigation-wise, cord blood is sent for SARS-CoV-2 serology at birth. Initially, nasopharyngeal swabs for SARS-CoV-2 RNA polymerase chain reaction (PCR) were performed in neonates after birth, and on days 1 and 2 of life, with accompanying stool samples sent for PCR. This has since been rationalised to only on days 1 and 2 of life. If the neonate is positive for SARS-CoV-2, venepuncture is performed for full blood count (FBC), liver function test (LFT), $\mathrm{C}$-reactive protein (CRP) and SARS-CoV-2 serology.

Baby A was born in good condition, via lower segment caesarean section at 38 weeks 6 days gestation, to an unvaccinated woman who developed symptomatic COVID-19 4 days prior to delivery. Mother's SARSCoV-2 nucleocapsid and spike antibody was negative. After birth, the baby was immediately separated from mother and formula milk-fed as per maternal wishes to prevent postnatal COVID-19 exposure.

The baby's nasopharyngeal SARS-CoV-2 RNA PCR at 2.5 hours, 24 hours and 51 hours of life were positive with decreasing CT values of 32.08, 19.57 and 13.63, respectively. Stool SARS-CoV-2 RNA PCR on the first and second day of life were positive (CT values 38.44 and 28.55, respectively). Blood for SARSCoV-2 RNA PCR and SARS-CoV-2 nucleocapsid and spike antibodies were negative. FBC, LFT and CRP were normal. Maternal placental histology was unremarkable. No maternal blood, placenta or amniotic fluid was sent for SARS-CoV-2 RNA PCR testing.

The baby remained asymptomatic and well throughout. He was reunited with mother on day 3 of life after SARS-CoV-2 infection was confirmed. Routine newborn care was maintained, and mother's personal decision was to continue with formula feeding. Birth hearing screen was delayed to 4 weeks later for infection control reasons. Routine blood spot screening for inborn errors of metabolism (IEM) was performed, and the IEM card was allowed to dry in baby's room before double-bagging for dispatch. Viral clearance was later observed with decreasing nasopharyngeal viral load (CT values were 16.55 and 23.25 on day 6 and day 10, respectively). On discharge, hand hygiene and droplet/contact precautions were emphasised, especially when handling diapers in case of prolonged stool shedding.

MIS-C in a healthy young child. Patient B is a Chinese boy who was 3 years 10 months old. He had 5 days of high fever $>40^{\circ} \mathrm{C}$ with classical features of Kawasaki disease-diffuse polymorphous rash, red cracked lips, non-suppurative conjunctivitis, bilateral cervical lymphadenopathy and puffy extremities. He also had abdominal pain, vomiting and poor oral intake. On initial examination, temperature was $37.9^{\circ} \mathrm{C}$, heart 
rate 148 beats/minute, respiratory rate 26/minute, and oxygen saturations $97 \%$ in room air. He was irritable and lethargic. He received fluid resuscitation of $30 \mathrm{~mL} /$ $\mathrm{kg}$ normal saline for evolving hypotension with a lowest blood pressure of $51 / 29 \mathrm{mmHg}$. In the next 8 hours, he subsequently developed multiorgan involvement of myocardial dysfunction with fluid-refractory cardiogenic shock and hepatomegaly, acute kidney injury, altered mental state, and biochemical evidence of hyperinflammation, lymphopaenia, thrombocytopaenia, coagulopathy and transaminitis.

Significant blood results were: lymphocytes $0.28 \times 10^{9} / \mathrm{L}$; platelets $63 \times 10^{9} / \mathrm{L}$; aspartate aminotransferase $52 \mathrm{U} / \mathrm{L}$; alanine transaminase $42 \mathrm{U} / \mathrm{L}$; urea $11.5 \mathrm{mmol} / \mathrm{L}$; creatinine $81 \mu \mathrm{mol} / \mathrm{L}$; prothrombin time 14 seconds; partial thromboplastin time $41.5 \mathrm{~s}$; D-Dimer $1.71 \mu \mathrm{g} / \mathrm{mL}$; fibrinogen $4.68 \mathrm{~g} / \mathrm{L}$; and peak lactate $7.08 \mathrm{mmol} / \mathrm{L}$. Inflammatory markers and cardiac biomarkers peaked on day 7 of illness (CRP 272mg/L, troponin 2,706ng/L and NT-pro-B-type natriuretic peptide $>35,000 \mathrm{pg} / \mathrm{mL}$ ) and subsequently declined (Table 1). Two-dimensional echocardiography done on day 6 of illness before inotropic support was started showed mildly impaired function with ejection fraction $45-53 \%$. There was no coronary artery involvement or pericardial effusion. Chest X-ray showed bilateral perihilar infiltrates, with no cardiomegaly or consolidation. Blood cultures, antistreptolysin antibody, and nasopharyngeal PCR for 13 respiratory pathogens (BioFire FilmArray RP2.1), including enterovirus/rhinovirus, adenovirus, influenza, respiratory syncytial virus, human coronavirus, metapneumovirus and mycoplasma pneumoniae, were negative.

Further investigations revealed previous recovered SARS-CoV-2 infection; SARS-CoV-2 nucleocapsidantibody reactive (74U/mL, Elecsys Roche), and SARSCoV-2 spike antibody $>250 \mathrm{U} / \mathrm{mL}$. Repeat confirmatory testing at National Public Health Laboratory also showed the presence of neutralising antibody $(92.03 \%$ inhibition value). COVID-19 PCRs from 2 nasopharyngeal swabs and 3 stool samples were negative. Investigations of parents, younger sibling and grandmother for serological and nasopharyngeal PCR evidence of acute or recovered infection were negative. The only known COVID-19 exposure was from a positive school contact 11 days prior to the start of illness. However, patient B had multiple negative surveillance PCR and antigen tests in the last 5 days prior to illness.

He required intensive care with dual inotropic and continuous positive airway pressure support for myocardial dysfunction. He was treated with empiric IV ceftriaxone $100 \mathrm{mg} / \mathrm{kg} /$ day, aspirin $5 \mathrm{mg} / \mathrm{kg} /$ day, intravenous immunoglobulin $2 \mathrm{~g} / \mathrm{kg}$ single dose, IV methylprednisolone $10 \mathrm{mg} / \mathrm{kg} /$ day for 4 days, and transitioned to weaning oral prednisolone. He also received enoxaparin and frusemide. IV ceftriaxone was stopped after 5 days when bacterial cultures remained negative and he demonstrated clinical improvement. There was biochemical improvement and almost

Table 1. Laboratory results of inflammatory and cardiac biomarkers by day of illness

\begin{tabular}{|c|c|c|c|c|c|c|c|}
\hline Test & Day 5 & Day 6 & Day 7 & Day 8 & Day 9 & Day 10 & Day 11 \\
\hline White blood cells, $\times 10^{9} / \mathrm{L}$ & 7.10 & 28.53 & 17.00 & 13.41 & 10.97 & 11.19 & 12.41 \\
\hline Neutrophils, $\times 10^{9} / \mathrm{L}$ & 6.13 & 26 & 15.26 & 10.59 & 7.92 & 7.69 & 9.66 \\
\hline Lymphocytes, $x 10^{9} / \mathrm{L}$ & 0.51 & 1.54 & 1.19 & 2.47 & 2.63 & 3.17 & 2.23 \\
\hline Haemoglobin, g/dL & 12.2 & 10.5 & 10.3 & 8.7 & 9.0 & 10.5 & 9.8 \\
\hline Platelets, $\times 10^{9} / \mathrm{L}$ & 63 & 89 & 67 & 62 & 59 & 78 & 107 \\
\hline C-reactive protein, $\mathrm{mg} / \mathrm{L}$ & 238 & NA & 272 & 245 & 125 & 71 & 31 \\
\hline Erythrocyte sedimentation rate, $\mathrm{mm} / \mathrm{hr}$ & 37 & NA & 143 & NA & NA & NA & NA \\
\hline Procalcitonin, $\mu \mathrm{g} / \mathrm{L}$ & NA & 21.67 & 22.95 & NA & 7.36 & NA & NA \\
\hline Ferritin, $\mu \mathrm{g} / \mathrm{L}$ & NA & 658 & 681 & 621 & 552 & 517 & 415 \\
\hline Creatine kinase, U/L & NA & 143 & 282 & 60 & 35 & NA & NA \\
\hline Creatine kinase $\mathrm{MB}, \mu \mathrm{g} / \mathrm{L}$ & NA & 24 & 15.2 & 6.3 & 3.4 & NA & NA \\
\hline Troponin I, ng/L & NA & 2857 & 3706 & 2814.0 & 1630 & 1108.0 & 706.1 \\
\hline NT-pro-B-type natriuretic peptide, $\mathrm{pg} / \mathrm{mL}$ & NA & $>35000$ & $>35000$ & $>35000$ & 23147 & 6718 & 2584 \\
\hline
\end{tabular}

NA: not available 
complete clinical resolution by day 10 of treatment. He was discharged on day 12 with outpatient follow-up arranged.

Discussion. Since the B.1.617.2 (otherwise known as "Delta") variant of SARS-CoV-2 virus emerged, it has been observed to exhibit greater transmissibility, shorter incubation periods, increased symptoms in afflicted persons and increased vaccine breakthrough infections. ${ }^{4}$ In our centre from 1 January to 17 September $2021,67.7 \%$ (21/31) of unvaccinated/partially vaccinated adolescents $>12$ years, and $76.3 \%(122 / 160)$ of children $<12$ years old were symptomatic, compared to $40 \%(2 / 5)$ and $34.4 \%(11 / 32)$, respectively in 2020. The latter is consistent with KK Women's and Children's Hospital that reported symptoms in $38.5 \%$ of children with COVID-19 infection admitted from January to May $2020 .{ }^{5}$

During the second wave, in addition to this increased proportion of symptomatic infected children, we observe a general trend that children who have fever tend to have longer durations of it, and sometimes a "saddleback" fever where the fever initially lyses and then returns for a few days, before resolving completely. Furthermore, with increased community spread, we started to receive severe COVID-19 paediatric cases requiring initiation of antiviral medications and steroids. We also had more children with high risk for deterioration due to underlying medical conditions such as malignancy or immunosuppression present with COVID-19 infection. Despite all this, almost all children have fully recovered with good outcomes.

Vertical in utero transmission and MIS-C are both rare with reported incidence of about $3-4 \% 6,7$ and $0.14 \%,{ }^{8}$ respectively. MIS-C, a hyperinflammatory syndrome occurring 2-10 weeks after SARS-COV-2 infection, mainly affects school-age children and is severe; two-thirds of cases require intensive care with a reported mortality of 2-4\%. ${ }^{8,9}$ Its symptomatology bears similarities with Kawasaki disease (KD) and toxic shock syndrome (TSS), as children present with fever that may be accompanied by rash, conjunctivitis, and mucosal changes. Compared with KD, children with MIS-C tend to manifest more gastrointestinal symptoms of abdominal pain, vomiting or diarrhoea. They also tend to exhibit greater haemodynamic compromise and multiorgan involvement than KD. TSS can present very similarly to MIS-C, hence the need for work-up and empiric antibiotic cover until bacterial infection is excluded. ${ }^{10}$ MIS-C has been reported worldwide, with racial and ethnic differences that are poorly understood.

As Singapore transitions to endemicity and unvaccinated children form an increasing proportion of the infected population, it is inevitable that rare and/or severe cases will arise. It is fortuitous that the 2 cases described here had good outcomes with no short-term sequelae. However, medium- to long-term outcomes of vertical transmission and MIS-C are unknown, with coronary artery aneurysmal changes in MIS-C posing a risk of significant long-term morbidity. ${ }^{11}$ Counselling of pregnant women with COVID-19 should focus on the greater risks of post-natal transmission, balanced with the need for early breastfeeding and mother-child bonding. Investigation of the newborn for in utero transmission should be routine. In addition, physicians should encourage pregnant women to get vaccinated to protect themselves as well as their unborn child, since it has been shown that being vaccinated earlier in the third trimester can result in high levels of anti-SARS-CoV-2 antibodies being placentally transferred to the fetus, hopefully providing passive immunity to the vulnerable newborn. ${ }^{12,13}$

As vaccinations are rolled out for younger children in Singapore, physicians will play an important role in educating parents on the risks and benefits of vaccination set against the likelihood of contracting COVID-19 and the possibility of suffering its complications of severe illness and MIS-C. Physicians should ensure parents of children who become infected are made aware of MIS-C and the clinical features of concern, which warrant early medical review.

\section{REFERENCES}

1. Ministry of Health Singapore. Media statement on paediatric MIS-C cases in Singapore, 6 November 2021. Available at: https://www. moh.gov.sg/news-highlights/details/media-statement-on-paediatric-misc-cases-in-singapore. Accessed on 3 December 2021.

2. Ministry of Health Singapore. COVID-19 statistics. Available at: https:// www.moh.gov.sg/covid-19/statistics. Accessed 3 December 2021.

3. Martins MM, Prata-Barbosa A, da CunhaAJLA. Update on SARS-CoV-2 infection in children. Paediatr Int Child Health 2021;41:56-64.

4. Grant R, Charmet T, Schaeffer L, et al. Impact of SARS-CoV-2 Delta variant on incubation, transmission settings and vaccine effectiveness: Results from a nationwide case-control study in France. Lancet Reg Health Eur 2022;13:100278.

5. Li J, Thoon KC, Chong CY, et al. Comparative Analysis of Symptomatic and Asymptomatic SARS-CoV-2 Infection in Children. Ann Acad Med Singap 2020;49:530-7.

6. Kotlyar AM, Grechukhina O, Chen A, et al. Vertical transmission of coronavirus disease 2019: a systematic review and meta-analysis. Am J Obstet Gynecol 2021;224:35-53.e3.

7. Chi J, Gong W, Gao Q. Clinical characteristics and outcomes of pregnant women with COVID-19 and the risk of vertical transmission: a systematic review. Arch Gynecol Obstet 2021;303:337-45.

8. Rubens JH, Akindele NP, Tschudy MM, et al. Acute Covid-19 and multisystem inflammatory syndrome in children. BMJ 2021; 372:n385.

9. Feldstein LR, Tenforde MW, Friedman KG, et al. Characteristics and outcomes of US children and adolescents with multisystem 
inflammatory syndrome in children (MIS-C) compared with severe acute COVID-19. JAMA 2021;325:1074-87.

10. Kabeerdoss J, Pilania RK, Karkhele R, et al. Severe COVID-19, multisystem inflammatory syndrome in children, and Kawasaki disease: immunological mechanisms, clinical manifestations and management. Rheumatol Int 2021;41:19-32.

11. Davies P, du Pré P, Lillie J, et al. One-year outcomes of critical care patients post-COVID-19 multisystem inflammatory syndrome in children. JAMA Pediatr 2021;175:1281-3.

12. Jorgensen SCJ, Burry L, Tabbara N. Role of maternal COVID-19 vaccination in providing immunological protection to the newborn. Pharmacotherapy 2022;42:58-70.

13. Rottenstreich A, Zarbiv G, Oiknine-Djian E, et al. Timing of SARS-CoV-2 vaccination during the third trimester of pregnancy and transplacental antibody transfer: a prospective cohort study. Clin Microbiol Infect 2021;S1198-743X:00601-7.
Olivia Min Yi Leow ${ }^{1}{ }_{M B B S}$, Rie $\underline{\text { Aoyama }}{ }^{1}{ }_{M B B c h}$, Si Min Chan ${ }^{1,2}{ }_{M B B S}$

${ }^{1}$ Division of Paediatric Infectious Diseases, Khoo Teck Puat-National University Children's Medical Institute, National University Hospital, Singapore

${ }^{2}$ Yong Loo Lin School of Medicine, National University of Singapore, Singapore

Correspondence: Dr Si Min Chan, Department of Paediatrics, Khoo Teck Puat-National University Children's Medical Institute, National University Hospital, 1E Kent Ridge Road, NUHS Tower Block, Level 12, Singapore 119228 .

Email: si_min_chan@nuhs.edu.sg 